\title{
Study on inhomogeneous perforation thick micro-perforated panel sound absorbers
}

\author{
Iwan Prasetiyo $^{1 *}$, Joko Sarwono ${ }^{1}$, Indra Sihar ${ }^{1}$ \\ ${ }^{1}$ Acoustic Laboratory, Engineering Physics, Institut Teknologi Bandung, \\ Ganesa 10 Bandung 40132 Indonesia, \\ *Email: i.prasetiyo@ fti.itb.ac.id \\ Phone:+62-22-2504424; Fax: +62-22-2506281
}

\begin{abstract}
Micro-perforated panel (MPP) sound absorbers are usually made of a thin panel and have narrow absorption bandwidth. This drawback causes the application of MPP to be limited. In this paper, the possibility of realizing wider absorption bandwidth MPP with sufficient structural strength is investigated. For this, multi-MPP (resonator) arranged in parallel to form an inhomogeneous perforation MPP is introduced to widen the absorption bandwidth. The thickness of MPP must be 1.5 times higher than perforation diameter or more in order to have appropriate strength. The characteristics of corresponding absorption coefficients are studied parametrically using theoretical models as thick panels can reduce the MPP's performance. It is found that the absorption bandwidth of thicker panels with inhomogeneous perforation approach can be at least twice times of classical MPP. The problem of reduced peak absorption coefficient in a thick panel can be avoided by keeping the acoustic resistance value around $1 \pm 0.5$ Rayls. Compared with homogeneous MPP, inter-resonator interaction exists in the inhomogeneous perforation thick MPP that causes the overall absorption to become higher due to the increasing of the acoustic resistance as well as the shifting of peak resonance following residual acoustic reactance. The measurement results confirm all of the characteristics.
\end{abstract}

Keywords: Micro-perforated panel absorber; thick panel; inhomogeneous perforation pattern.

\section{INTRODUCTION}

As an alternative acoustic absorber to traditional porous materials, the applications of Micro-perforated panels (MPP) can be found in various fields such as room acoustics [1-3], environmental noise abatement [4], noise control [5], and etc. Dah-You [6] formulated the MPP on the basis of the Helmholtz resonance mechanism. The sound absorption mechanism leads to narrower absorption frequency range unlike the porous materials. Apart from this, the ratio of perforation diameter and the panel thickness should also be nearly one for optimum design [7]. Hence, MPP is usually made of a thin panel which is less than $1 \mathrm{~mm}$ thick as perforation diameter of MPP must be less than 1 $\mathrm{mm}$. The two facts cause the MPP to not always be applicable for practical purposes e.g. for the case of the interior finish of room walls where more physical resistance and wider sound absorption bandwidth are commonly required to deal with preferred sound fields such as the acoustic characteristics in mosques [8]. 
Compared with the classical MPP, the use of thicker panels for MPP has potential to reduce absorption performance in terms of amplitude and frequency bandwidth due to increasing acoustic resistance impedance and/ or reactance [9]. In principle, a wider absorption bandwidth MPP will need larger acoustic resistance and smaller acoustic reactance [7,9] but larger acoustic resistance can reduce the absorption amplitude. Hence, great care is required to introduce thicker panels to MPP. To deal with that, some studies have been done by modifying the form of pores in order to improve the performance e.g. tapered holes $[9,10]$ for micro-perforated insertion units (MIU) [11]. Even though that can deal with the thicker panel effect on MPP but it still poses a difficulty from the practical point of view e.g. manufacturing cost and technology. Meanwhile, some studies have been proposed specifically regarding widening absorption bandwidth. Jung et al. [12] focused to develop double and triple layer systems to obtain multiple absorption peaks in which a wider absorption bandwidth of more than 4 octaves can be found accordingly. A similar approach can also be found from the study conducted by Sakagami et al. [13] from which a detailed analysis with Helmholt-Kirchhoff is provided. This approach outperformed the single layer but more space was required to implement such constructions which is not also always applicable in practice. Moreover, the relation between the acoustic impedance of each sub-system is not clear. Hence, an optimization technique is not easily applied. Additionally, another effort is put by arranging multiple conventional MPP in parallel form [14] while the backing air cavity is partitioned. Multiple peaks can be obtained from such system and a wider absorption can be obtained accordingly. Recently, Qian et al. [15] did an experimental investigation on the effect of reducing the perforation diameter of MPP to less than $100 \mu \mathrm{m}$. It was found that half-absorption bandwidth of 3-4 octaves with the peak absorption higher than 0.85 were pronounced. However, such approach is not always a good option as it requires special manufacturing technology e.g. using MEMS technology. More recently, a more complex construction of MPP incorporating panel absorber is also proposed to deal with a wider absorption bandwidth by enhancing low frequency absorption[16].

The work in this paper focuses on investigating the possibility of having wider absorption bandwidth for thick MPP. The thick MPP is considered when the panel thickness $t$ is greater than the perforation diameter $d(t>d)$. The effect of $t$ on acoustic impedance and their interdependency are discussed in terms of absorption performance and absorption frequency bandwidth. Subsequently, inhomogeneous perforation is introduced on thick panels in order to get wider absorption bandwidth and the corresponding characteristics are compared to that of the homogeneous MPP.

\section{METHODS AND MATERIALS}

\section{Impedance Model for Micro-perforated Panels}

Dah-You [6] first proposed an approximate model to calculate sound absorption of the micro-perforated panel by treating the absorber consisting of parallel connected tubes distributed over surface and the panel is considered rigid. The approximate model was developed by simplifying the Bessel function. For normal incidence, the wave motion in all the short tubes can be regarded to be in phase and additive. Therefore, the relative acoustic impedance with considering the end correction, this yields [7] 


$$
z=z_{r}+j z_{i m}=\frac{32 \eta t}{\sigma \rho_{0} c d^{2}}\left(\left[1+\frac{k^{2}}{32}\right]^{1 / 2}+\frac{\sqrt{2}}{32} k \frac{d}{t}\right)+j \frac{\omega t}{\sigma c}\left(1+\left[1+\frac{k^{2}}{2}\right]^{-1 / 2}+0.85 \frac{d}{t}\right)
$$

The perforation constant $k=d \sqrt{\omega \rho_{0} / 4 \eta}$ where $d$ is the perforation diameter, $\omega$ is the angular frequency, $\rho_{0}$ is the air density, $\eta$ is the coefficient of fluid viscosity, and the perforation ratio area with circular cross section $\sigma=(\pi / 4)(d / b)^{2}$ where $b$ is the centreto-centre distance between holes. Moreover, the term $z_{r}$ is responsible for the resistance component of acoustic impedance, while the term $z_{i m}$ is for acoustic reactance.

MPP absorbers require a backing air cavity with cavity depth $D$. It is required to tune its absorption at resonance frequency $f_{0}$ at which the maximum absorption can be obtained. The air cavity impedance is expressed as

$$
z_{c a v}=-j \cot \frac{\omega D}{c}
$$

The combined surface impedance of the MPP and the air in the cavity $z_{m p p}$ is thus given by

$$
z_{m p p}=z-j \cot \frac{\omega D}{c}
$$

For normal incidence, the sound absorption coefficient is thus defined as

$$
\alpha_{0}=\frac{4 z_{r}}{\left(1+z_{r}\right)^{2}+\left(z_{i m}-\cot \omega D / c\right)^{2}}
$$

and the maximum coefficient is given by

$$
\alpha_{\max }=\frac{4 z_{r}}{\left(1+z_{r}\right)^{2}}
$$

The multiple resonance frequencies $f_{0}$ are introduced by the following terms

$$
z_{\text {im }}-\cot (\omega D / c)=0
$$

For an acoustic compact condition, the expression of Eq. (6) is analogous to an ordinary mass-spring oscillator with the mass corresponding to $z_{i m}$ and the spring corresponding to $\cot (\omega D / c)$.

\section{Inhomogeneous Perforation Pattern Model}

The inhomogeneous perforation pattern in MPP is realized using a combination of multiple MPPs with different parameters and parallel to each other with the cavity partitioned as shown in Figure 1 in which its electrical equivalent model can also be observed. 

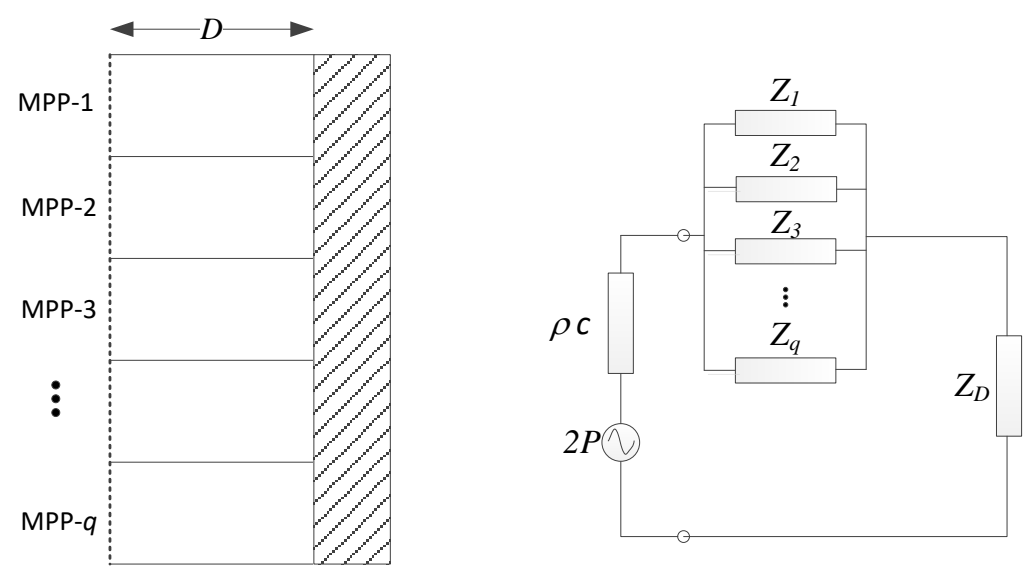

Figure 1. Inhomogeneous perforation pattern MPP arrangement (side view) and its electrical equivalent model

To predict the absorption coefficient of such system, the surface impedance $Z_{m p p}$ in Eq. (3) is modified to include the effect of different perforation parameters on the sound absorption. Using electro-acoustic equivalent, the overall surface impedance of MPP can be regarded as parallel composition of single MPP. Hence, the impedance over entire surface $\bar{Z}_{m p p}$ can be derived statistically as follows

$$
\frac{1}{\bar{Z}_{m p p}}=\sum_{i=1}^{q} a_{q} \frac{1}{Z_{m p p, q}}
$$

where $q$ is the number of MPP on the same panel surface, and $a_{q}=A_{q} / A_{T}$ is ratio area of the sub-MPP to the total area. Therefore, the combined surface impedance of the MPP and the air in the cavity $z_{m p p}$ can be expressed as

$$
Z_{m p p}=\bar{Z}_{m p p}-j \cot \frac{\omega D}{c}
$$

In which the air cavity depth $D$ is considered to be the same for all the sub-MPP. Subsequently, the absorption coefficient can be obtained using Eq. (4). It should be noted that the cavity needs to be partitioned rather than connected for all MPP to enable the sub-MPP to work individually. Actually, the surface impedance expressed by Eq. (7) does not include the effect of discontinuity of acoustic impedance that is present due to two different adjacent MPP. Such discontinuity can introduce excess absorption. Hence, the formulation is extended to incorporate the wave scattering on panel surface using the following expression [17].

$$
\alpha=1-\sum_{\operatorname{Re}\left[\gamma_{m}\right]}\left|\boldsymbol{\Psi}_{m}\right|^{2} \frac{\gamma_{m}}{\gamma_{0}}
$$

where $\gamma_{0}=\kappa \cos \theta$ with, $\kappa=2 \pi / \lambda$, and $\theta$ the incident angle (normal incidence angle $\left.\theta=0^{\circ}\right), \gamma_{m}=\sqrt{\kappa^{2}-\alpha_{m}^{2}}$, and $\boldsymbol{\Psi}_{m}$ is unknown wave scattering coefficient. 


\section{PARAMETRIC SURVEY}

\section{Effect of Panel Thickness}

Figure 2 presents the effect of panel thickness on the absorption coefficient for the same cavity depth with properties listed in Table 1 unless otherwise stated. The MPP is categorized as the homogenous perforation MPP. It can be seen that the peak frequency shifts to lower frequency with increased thickness This situation can be explained in accordance with Eq. (6). The thicker panels have greater total mass of air inside the perforation compared with that of the $1.5 \mathrm{~mm}$ thick panel while the stiffness of resonator system is unchanged due to the same air cavity depth. Hence, the resonant frequency related to that frequency peak becomes lower.

Table 1. Properties of micro-perforated panel.

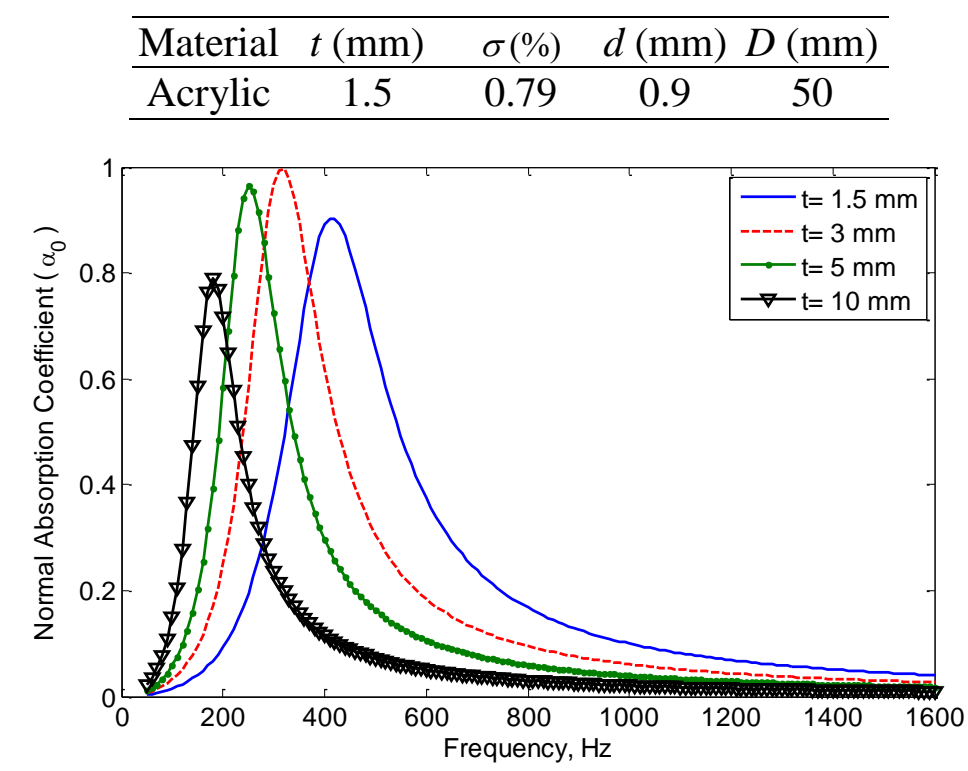

Figure 2. Absorption coefficient comparison for different panel thickness $t$ with air cavity depth $D=50 \mathrm{~mm}$

Table 2. Absorption and acoustic impedance characteristics of homogeneous MPP for different panel thickness.

\begin{tabular}{cccccc}
\hline$t(\mathrm{~mm})$ & $f_{0}(\mathrm{~Hz})$ & $\alpha_{\max }$ & $\begin{array}{c}\text { half-absorption } \\
\text { bandwidth }(\mathrm{Hz})^{*}\end{array}$ & $z_{r}$ & $z_{\text {im }}$ \\
\hline 1.5 & 414.5 & 0.90 & 223 & 0.523 & 2.511 \\
3 & 317.5 & 1.00 & 187 & 0.931 & 3.351 \\
5 & 253.2 & 0.97 & 149 & 1.447 & 4.240 \\
10 & 181.8 & 0.79 & 89 & 2.683 & 5.963 \\
\hline
\end{tabular}

*frequency bandwidth evaluated at $\alpha \geq 0.5$

Considering the thickness $t$ in Eq. (1), this parameter affects the resistance and reactance part of acoustic impedance. The maximum absorption requires the resistance component $z_{r}$ to be close to 1 as indicated by Eq. (5). Meanwhile, the absorption 
bandwidth is more wide for smaller acoustic reactance $z_{i m}$. From Table 2, it is clear that the MPP with $z_{r}$ close to 1 has the higher absorption coefficient. Likewise, it can also be seen that the acoustic reactance $z_{\text {im }}$ of MPP increased as the panel thickness increased so that the associated bandwidth becomes narrower.

The results suggest that it is important to find the balance between the value of acoustic resistance and reactance in the design process in order to maintain the MPP's performance as both components are influenced by the panel's thickness. It is found from parametric survey that the value of acoustic resistance $z_{i}$ close to $1 \pm 0.5$ causes the MPP panel to have reasonable coefficient absorption $(\alpha=0.9-1)$, while the smaller or higher acoustic resistance than that value will bring to lower absorption coefficient. Meanwhile, acoustic reactance $z_{\text {im }}$ that is greater than 4 will lead to narrower bandwidth hence it is only effective for specific noise control e.g. tonal noise problem. Controlling the perforation rate $\sigma$ can be useful for the thick panel as long as the target frequency range is not the main concent. It can be seen from Figure 3 that the thick panel will be outperformed by the thinner one as this is dependent on the ratio of $(b / d)$ as shown in Figure 3. It is found that in order to have reasonable absorption coefficient for thick panel, the perforation ratio area need to be kept high. Hence, Eq. (5) reduces to

$$
\alpha_{\max } \approx \frac{4 \sigma}{t}=\frac{5.1}{t}\left(\frac{b}{d}\right)^{2}
$$

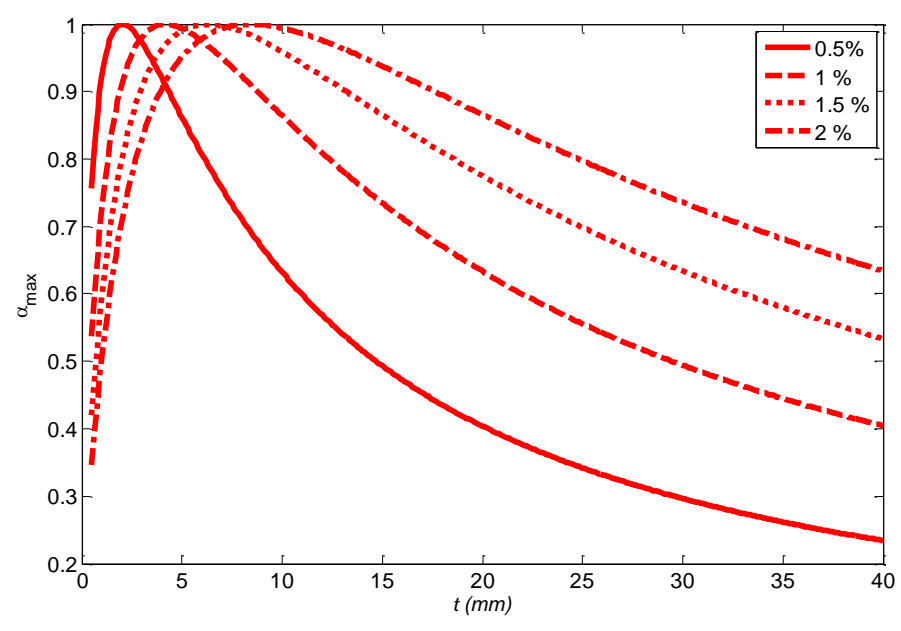

Figure 3. Effect of thickness on the maximum absorption coefficient and perforation area ratio.

\section{Effect of Inhomogeneous Perforation Pattern}

Inhomogeneous perforation pattern is considered as the results of the combination of two sub-MPPs. The perforation parameters are kept the same (see Table 1) except the distance between holes $b$ is varied so that the perforation ratio area $\sigma$ of each MPP varies. It can be seen from

Figure 4 that the overall half-absorption frequency bandwidth becomes wider for larger $b_{\text {ratio }}=\left(b_{1} / b_{2}\right)$ which is almost twice that of individual MPP as indicated for the case of $b_{\text {ratio }}$ of 2 . Care must be taken as the wider bandwidth is able to sacrifice the absorption amplitude. Hence, this approach has potential to widen the absorption bandwidth. 

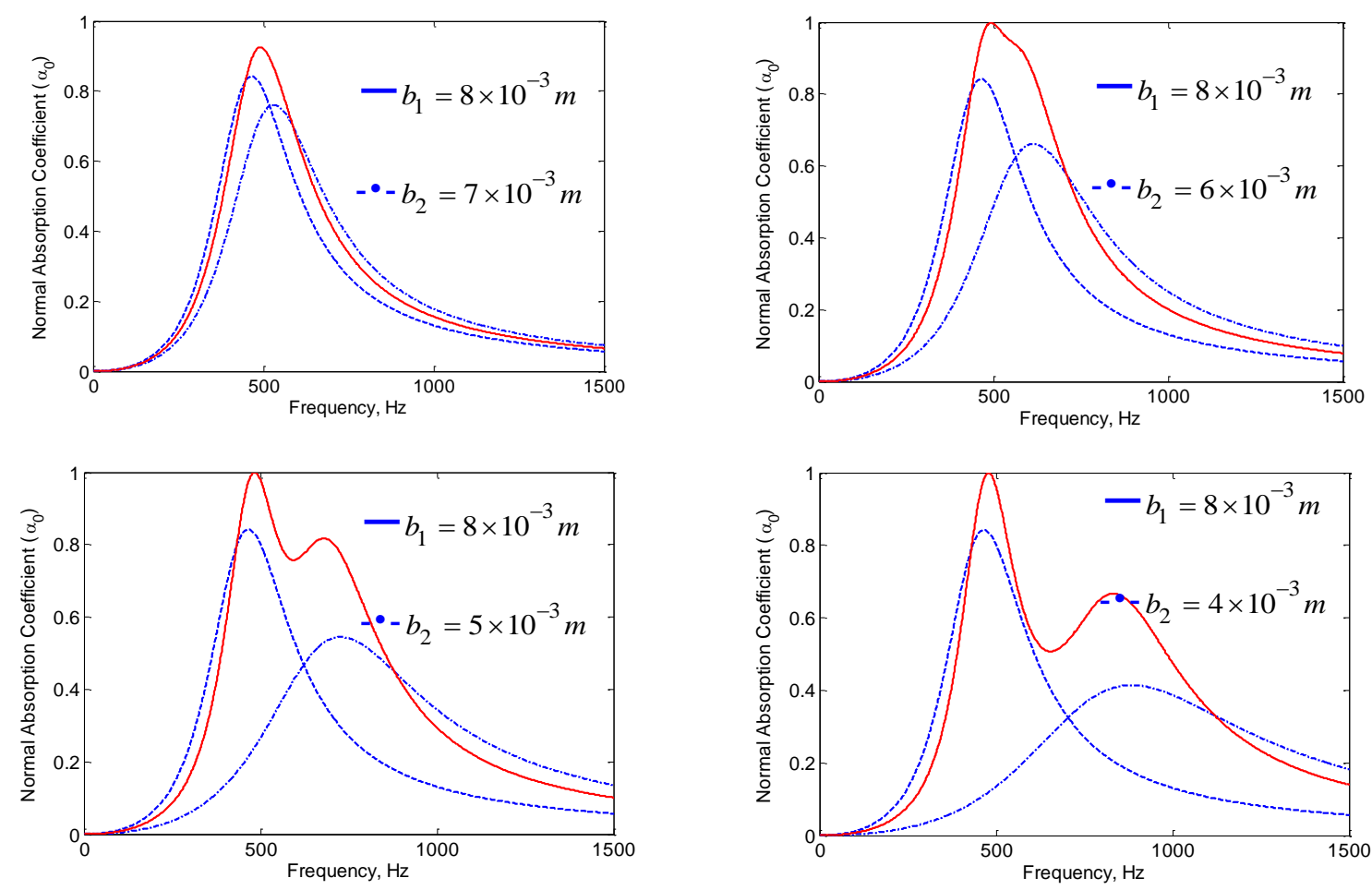

Figure 4. Effect of inhomogeneous perforation pattern with two sub-MPPs on the normal absorption coefficient. The red solid line indicates the overall normal absorption coefficient due to both sub-MPPs.

Table 3. Half frequency bandwidth absorption comparison for different thickness

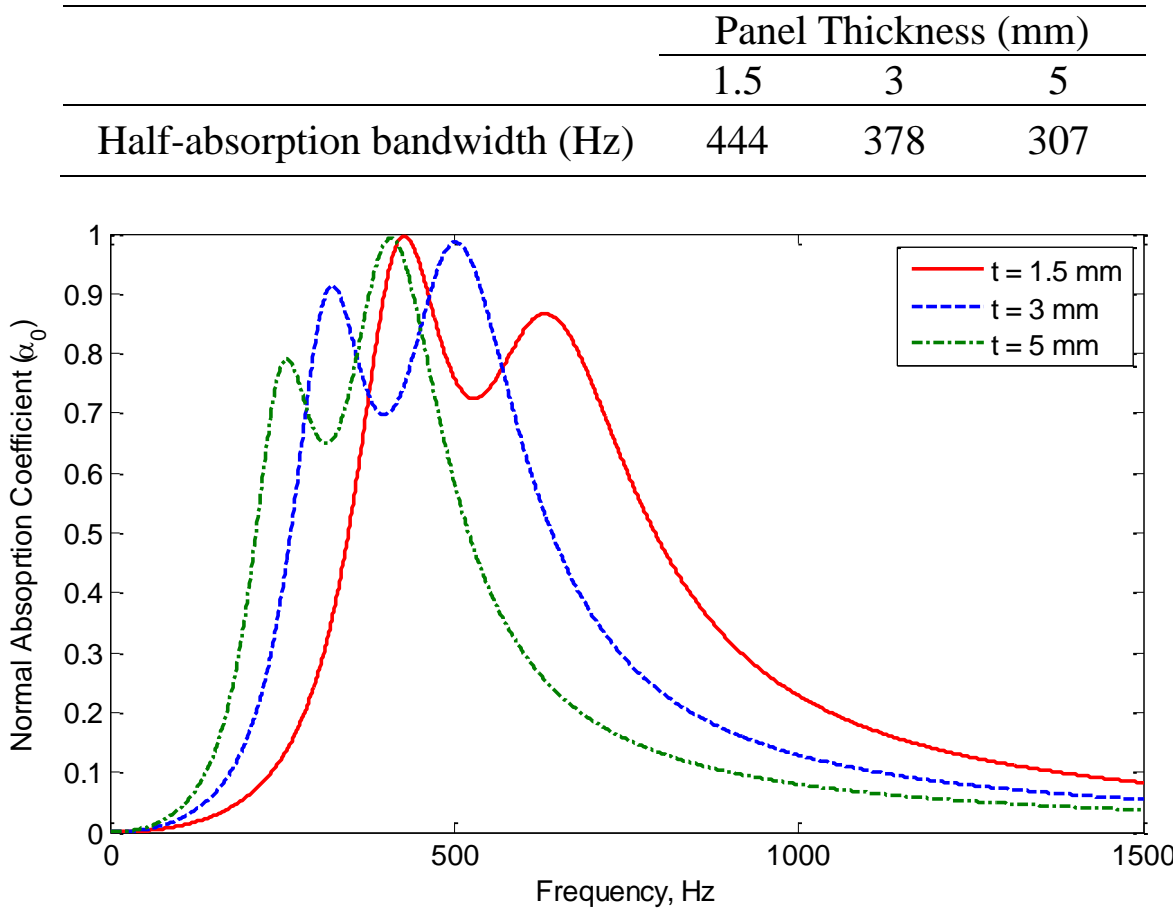

Figure 5. MPP absorption coefficient comparison for different thickness as a result of combination MPP $1(\sigma=0.79 \%)$ and MPP2 $(\sigma=2.08 \%)$ with cavity depth $50 \mathrm{~mm}$. The rest of the parameters are kept the same 
It is instructive to have a look at the absorption characteristic of inhomogeneous perforation pattern on thicker panels. The results can be observed from Figure 5. It is clear that the absorption bandwidth gets wider compared with the homogeneous ones for the same panel thickness. As indicated inTable 3, the half-absorption bandwidth are $444 \mathrm{~Hz}, 378 \mathrm{~Hz}$, and $307 \mathrm{~Hz}$ for panel thickness $1.5 \mathrm{~mm}, 3 \mathrm{~mm}$, and $5 \mathrm{~mm}$ respectively. The bandwidth are twice wider than the homogenous perforation MPP (see Table 2). Moreover, the maximum absorptions are in the range of 0.79 up to 0.99 . Hence, the MPP is still useful for absorber material. Despite this, the effect of thickness on absorption characteristic is still present i.e. the absorption coefficient decreased and the bandwidth is reduced for thicker panels e.g. for the case of the $1.5 \mathrm{~mm}$ thick MPP and the $5 \mathrm{~mm}$ thick MPP.

\section{RESULTS AND DISCUSSION}

\section{Experimental Setup}

For experimental work, the basic specification of micro-perforated specimens are made of $1.5 \mathrm{~mm}$ thick acrylic with following properties: Young's modulus, $E 3.2 \times 10^{9} \mathrm{~N} / \mathrm{m}^{2}$, Poisson's ratio, $v_{p} 0.35$, and density $\rho 1.16 \times 10^{3} \mathrm{~kg} / \mathrm{m} 3$. Meanwhile, the pore diameter of specimen is $0.9 \mathrm{~mm}$ and the air cavity depth is $50 \mathrm{~mm}$ unless otherwise stated, i.e. specimen for investigation of the effect of inhomogeneous perforation. The design parameters of the MPP are listed in Table 4. It should be noted that the inhomogeneous perforation pattern is realized by combining two MPPs designated as MPP-1 and MPP-2 with arrangement as shown in Figure 1.

Table 4. Geometrical properties of specimens.

\begin{tabular}{ccccc}
\hline Specification & Specimen & $t(\mathrm{~mm})$ & $D(\mathrm{~mm})$ & $\sigma(\%)$ \\
\hline \multirow{2}{*}{ Varying thickness } & 1 & 1.5 & 50 & 0.79 \\
& 2 & 3 & 50 & 0.79 \\
& 3 & 5 & 50 & 0.79 \\
\hline \multirow{2}{\text{Inhomogeneousperforation}}{} & 4 & 1.5 & 17 & 0.79 \\
pattern & 5 & 3 & 17 & 2.08 \\
& & & 17 & 0.79 \\
& & 5 & 17 & 0.79 \\
& & 5 & 17 & 2.08 \\
\hline
\end{tabular}

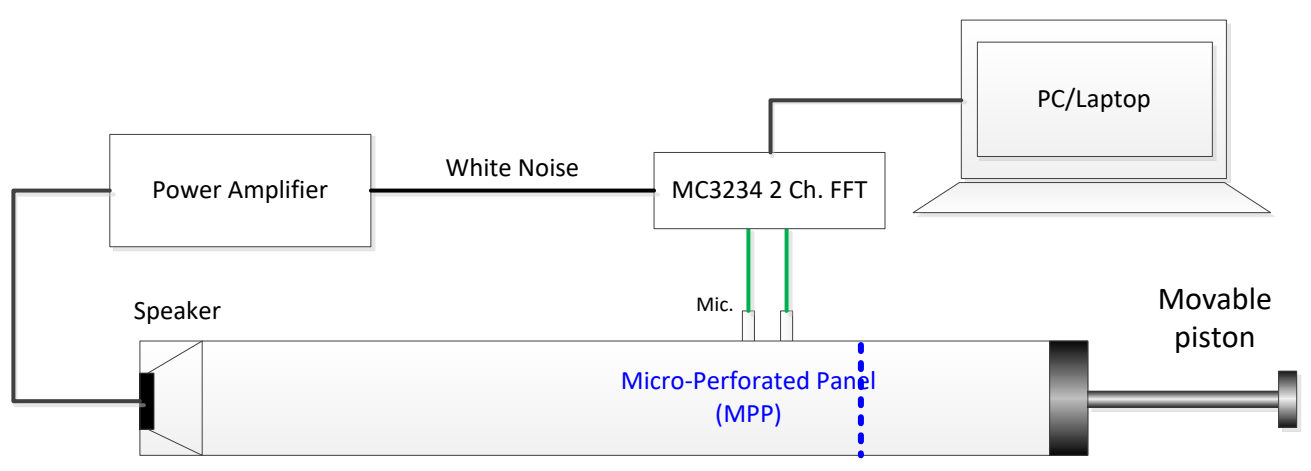

Figure 6. Schematic diagram of impedance tube test method. 
The measurement of absorption coefficient of MPP was conducted using impedance tube as per ISO 10534-2 [18]. The schematic diagram of the test can be seen in Figure 6. In principle, the white noise was generated in sound source and the travelling plane waves through a $10 \mathrm{~cm}$ radius tube were picked up using two microphones. From this, the transfer impedance can be determined and the sound absorption coefficient for frequency ranging from $64 \mathrm{~Hz}$ to $1.6 \mathrm{kHz}$ can be obtained accordingly.

\section{Effect of Panel Thickness}

The comparison results as shown in Figure 7 indicate that the predicted results and measured ones have similar tendency (specimens 1, 2, and 3). Moreover, the peak frequencies of the measured ones are close to the theoretical model (Maa model) which correspond with Helmholtz resonance. The discrepancy of the Maa model results and the measurement ones are around $4 \%$ up to $14 \%$ at half absorption frequency range. Some peaks are also present that are related with the panel resonances [19, 20]. Those peaks are not considered in the Maa model so they are missing from the predicted results. The issue of absorption bandwidth found in the parametric survey where the thicker panel has narrower bandwidth is also confirmed by the measurement results. Considering the comparison results, it is possible to have thick MPP with reasonable performance which is useful for practical purposes.

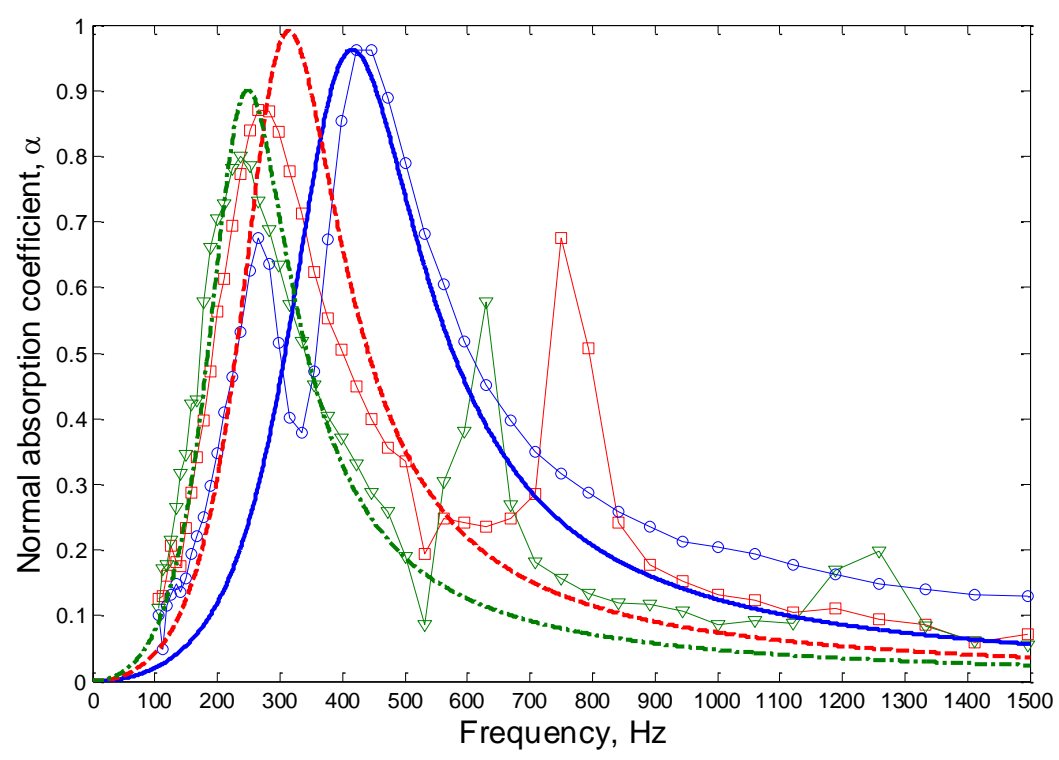

Figure 7. Absorption coefficient comparison of Maa' model and measurement for different panel thickness (Maa model: $-t=1.5 \mathrm{~mm} ;---t=3 \mathrm{~mm} ;-\bullet-t=5 \mathrm{~mm}$; measurement results $-\mathrm{o}-t=1.5 \mathrm{~mm} ;-\square-t=3 \mathrm{~mm} ;-\nabla-t=5 \mathrm{~mm}$ )

\section{Effect of Inhomogeneous Perforation Pattern}

Figure 8 (a) presents normal absorption coefficient comparison of prediction results and measurement results for inhomogeneous perforation MPP (specimen 4). It is clear that two absorption peaks are pronounced from the measurement results at $766 \mathrm{~Hz}$ and 1122 
$\mathrm{Hz}$ as indicated by the blue circle. Compared with the homogeneous perforation pattern MPP-1 and MPP-2, see the green dashed lines, the inhomogeneous pattern is apparently formed by two individual MPP as expected. The analytical results, particularly calculated using Eq. (8), is in good agreement with the measurement results except the presence of gap in frequency of around $841 \mathrm{~Hz}$ up to $1059 \mathrm{~Hz}$. This gap exists as the effective perforation area is reduced by $1 / 2$ of the original value considering the nonresonating MPP behaves as a rigid wall. Hence, the acoustic resistance is increased by two times. The effect of finite specimen size is also present as the analytical model particularly Eq. (10) is developed using periodic condition for infinite system so that its result is less accurate compared with that of Eq. (8). A similar situation is found for the case of $3 \mathrm{~mm}$ thick MPP and $5 \mathrm{~mm}$ thick MPP respectively [see Figure 8 (b) and (c)]. Moreover, the analytical models employed in this paper are still useful to predict the absorption coefficient of inhomogeneous perforation thick MPP. Meanwhile, the presence of the peak seen at $502 \mathrm{~Hz}$ corresponds to fundamental mode of the panel rather than was caused by the Helmholtz resonance.

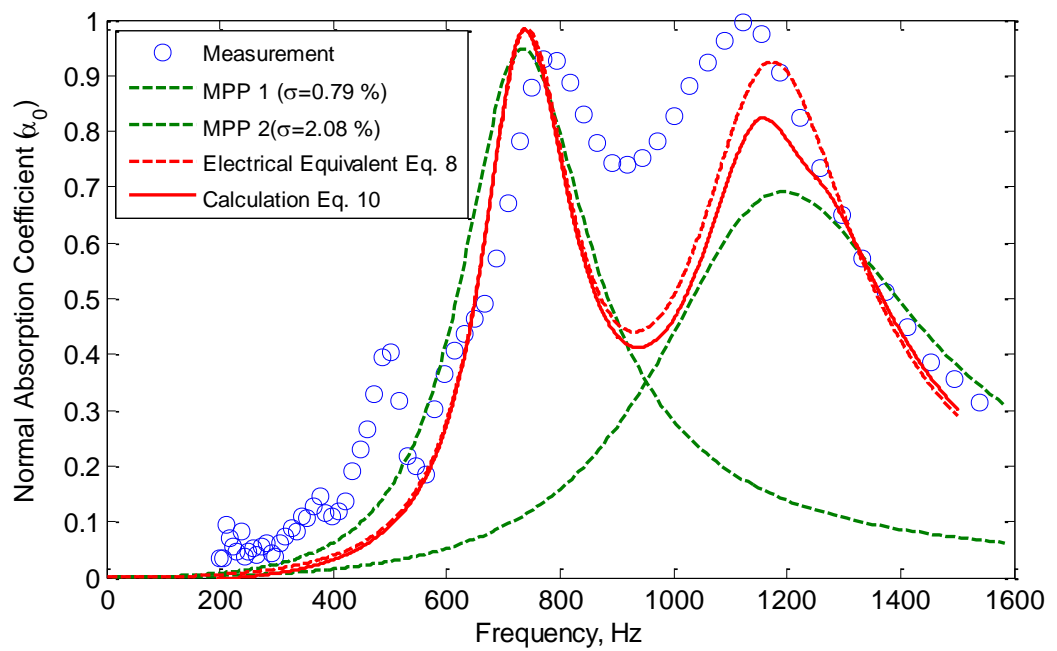

(a)

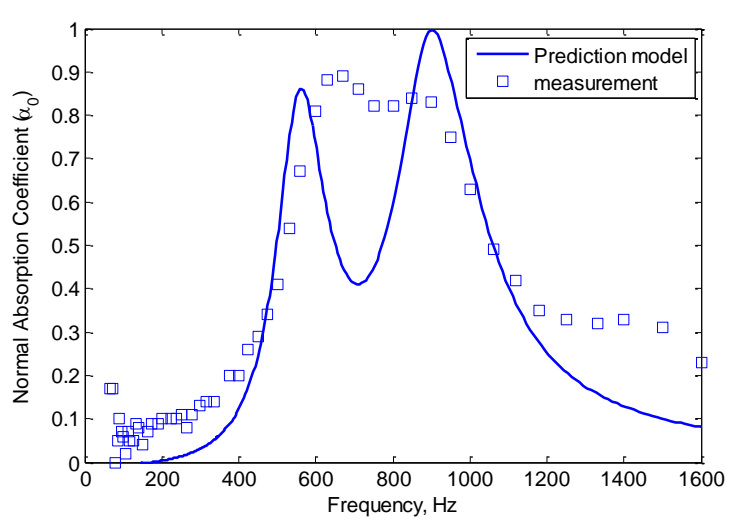

(b)

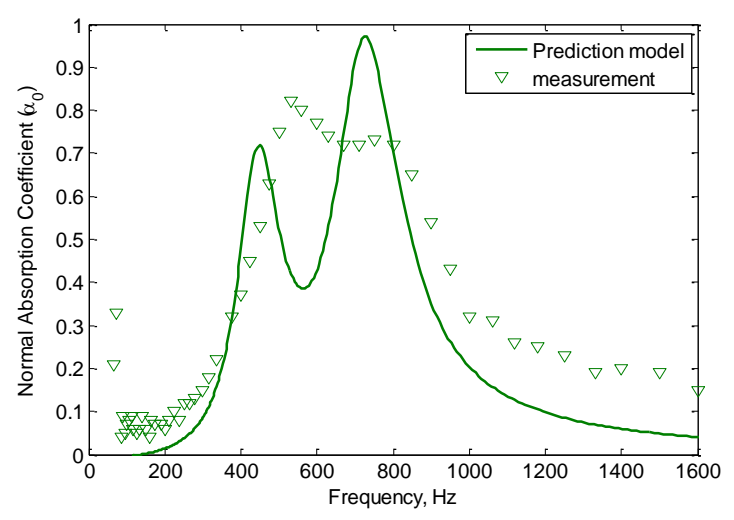

(c)

Figure 8. Normal absorption coefficient of inhomogeneous perforation comparison of measurement results and analytical ones: (a) $1.5 \mathrm{~mm}$ thick MPP; (b) $3 \mathrm{~mm}$ thick MPP; and (c) $5 \mathrm{~mm}$ thick MPP

It is also interesting to see that the corresponding peaks do not perfectly match compared with individual MPP or even for inhomogeneous perforation analytical model 
results. Actually, the two Helmholtz resonator systems in the inhomogeneous perforation MPP work individually rather than simultaneously. However, considering the two MPP are laid on the same surface, the acoustic reactance of the non-resonating MPP will be negative or positive. It can be seen from Figure 9 from which the acoustic reactance of impedance of two MPPs is compared. The peak frequency of MPP-1 shifts to higher frequency as residual of $\operatorname{Im}\left(Z_{m p p}\right)=z_{i m}-\cot (\omega D / c)<0$. This condition imposed an added-stiffness effect. Coversely, the peak frequency of MPP-2 shifts to lower frequency as residual of $\operatorname{Im}\left(Z_{m p p}\right)=z_{i m}-\cot (\omega D / c)>0$ so that an added-mass effect is present. Hence, there is what is so called as inter-resonator interaction contributing to the overall absorption coefficient.

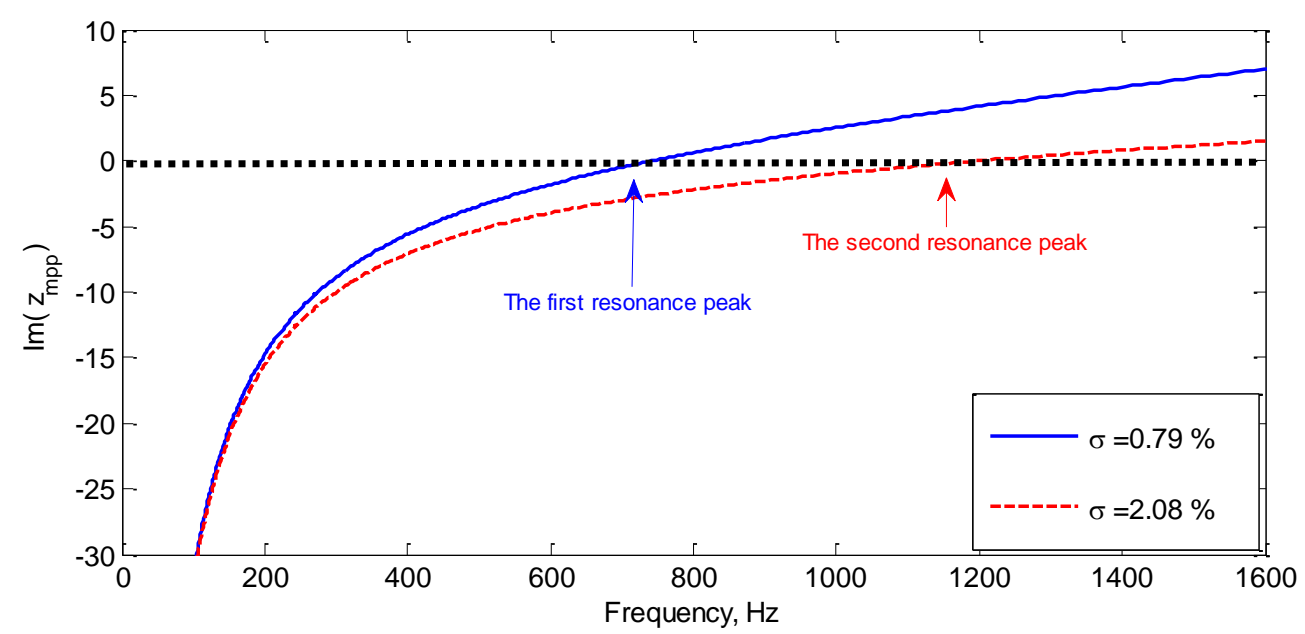

Figure 9. Acoustic reactance characteristic of MPP-1 $(\sigma=0.789 \%)$ and MPP-2

$$
(\sigma=2.08 \%)
$$

\section{CONCLUSIONS}

The characteristic of inhomogeneous perforation pattern on thick MPP has been studied. A thick MPP experiences higher acoustic resistance as well as acoustic reactance compared with the classical one where the ratio of perforation diameter and panel thickness is nearly one to obtain an optimal design. For maximum absorption coefficient, it is important to keep the acoustic resistance value around $1 \pm 0.5$ Rayls. Meanwhile, the absorption bandwidth can be widened through the inhomogeneous perforation approach as the reduction of absorption bandwidth cannot be avoided for thicker panels. This can lead to at least twice times homogeneous MPP.

It is also found that inter-resonator interaction contributed to the overall absorption coefficient in the inhomogeneous perforation MPP. Compared with homogeneous MPP, this causes the overall absorption to become higher due to the increasing of the acoustic resistance as well as the shifting of peak resonance due to residual acoustic reactance. The theoretical model based on statistical model and wave scattering can produce reasonable results for prediction purposes. After testing the MPP specification with thickness up to $5 \mathrm{~mm}$, it is still possible to have more applicable MPP where more resistance to physical contact and wider absorption bandwidth are usually required. Moreover, the use of conventional circular perforation in the inhomogeneous perforation thick MPP can help to manufacture the MPP in a more simpler way. 


\section{ACKNOWLEDGEMENTS}

The research was funded by the ITB's Research Innovation Grant under project number 144.23/AL-1/DIPA/PN/SPK/2013

\section{REFRENCES}

[1] Fuchs HV, Zha X. Acrylic-glass sound absorbers in the plenum of the deutscher bundestag. Applied Acoustics. 1997;51:211-7.

[2] Sarwono J, Prasetiyo I, S Andreas, William A. The Design of MPP and its Application to Enhance the Acoustics of a Real Auditorium. Inter-Noise 43rd International Congress on Noise Control Engineering. Melbourne2014.

[3] Fuchs HV, Zha X. Micro-Perforated Structures as Sound Absorbers \&\#8211; A Review and Outlook. Acta Acustica united with Acustica. 2006;92:139-46.

[4] Asdrubali F, Pispola G. Properties of transparent sound-absorbing panels for use in noise barriers. The Journal of the Acoustical Society of America. 2007;121:214-21.

[5] Yu X, Cheng L, You X. Hybrid silencers with micro-perforated panels and internal partitions. The Journal of the Acoustical Society of America. 2015;137:951-62.

[6] Dah-You M. Theory and design of microperforated panel sound-absorbing constructions. Scientia Sinica. 1975;18:55-71.

[7] Maa D-Y. Potential of microperforated panel absorber. The Journal of the Acoustical Society of America. 1998;104:2861-6.

[8] Kassim DH, Putra A, Nor MJM, Muhammad N. Effect of pyramidal dome geometry on the acoustical characteristics in a mosque. Journal of Mechanical Engineering and Sciences. 2014;7:1127-33.

[9] Sakagami K, Morimoto M, Yairi M, Minemura A. A pilot study on improving the absorptivity of a thick microperforated panel absorber. Applied Acoustics. 2008;69:179-82.

[10] Randeberg RT. Perforated panel absorbers with viscous energy dissipation enhanced by orifice design: Norwegian University of Science and Technology, Trondheim; 2000.

[11] Pfretzschner J, Cobo P, Simon F, Cuesta M, Fernández A. Microperforated insertion units: An alternative strategy to design microperforated panels. Applied Acoustics. 2006;67:62-73.

[12] Jung SS, Kim YT, Lee DH, Kim HC, Cho SI, Lee JK. Sound absorption of micro-perforated panel. Journal-Korean Physical Society. 2007;50:1044.

[13] Sakagami K, Matsutani K, Morimoto M. Sound absorption of a double-leaf micro-perforated panel with an air-back cavity and a rigid-back wall: Detailed analysis with a Helmholtz-Kirchhoff integral formulation. Applied Acoustics. 2010;71:411-7.

[14] Sakagami K, Nagayama Y, Morimoto M, Yairi M. Pilot study on wideband sound absorber obtained by combination of two different microperforated panel (MPP) absorbers. Acoustical Science and Technology. 2009;30:154-6.

[15] Qian Y, Kong D, Liu S, Sun S, Zhao Z. Investigation on micro-perforated panel absorber with ultra-micro perforations. Applied Acoustics. 2013;74:931-5. 
[16] Zhao X, Fan X. Enhancing low frequency sound absorption of micro-perforated panel absorbers by using mechanical impedance plates. Applied Acoustics. 2015;88:123-8.

[17] Takahashi D. Excess sound absorption due to periodically arranged absorptive materials. The Journal of the Acoustical Society of America. 1989;86:2215-22.

[18] Doutres O, Salissou Y, Atalla N, Panneton R. Evaluation of the acoustic and non-acoustic properties of sound absorbing materials using a three-microphone impedance tube. Applied Acoustics. 2010;71:506-9.

[19] Lee Y, Lee E, $\mathrm{Ng} \mathrm{C}$. Sound absorption of a finite flexible micro-perforated panel backed by an air cavity. Journal of Sound and Vibration. 2005;287:227-43.

[20] Bravo T, Maury C, Pinhède C. Vibroacoustic properties of thin micro-perforated panel absorbers. The Journal of the Acoustical Society of America. 2012;132:789-98. 